\title{
Reduction of dislocation density of aluminium nitride buffer layer grown on sapphire substrate
}

\author{
K. Jesbains ${ }^{1}$, N. Kuwano ${ }^{1}$, K.R Jamaludin ${ }^{1}$, H. Miyake ${ }^{2}$, K. Hiramatsu ${ }^{2}$, S. \\ Suzuki $^{2}$, M. Mitsuhara ${ }^{3}$ S.Hata $^{3}$ and Y.Soejima ${ }^{3}$ \\ ${ }^{1}$ Malaysia-Japan International Institute of Technology, UTM, Kuala Lumpur \\ ${ }^{2}$ Dept. Electrical and Electronic Engineering, Mie University, Tsu Japan \\ ${ }^{3}$ Graduate School of Engineering Sciences, Kyushu University, Kasuga Japan \\ *Email: jesbains5@gmail.com \\ Phone: +60126644958
}

\begin{abstract}
An aluminium nitride (AlN) buffer layer with $200 \mathrm{~nm}$ thickness was grown on (0001) sapphire substrate using the metal-organic vapour phase epitaxy (MOVPE) method in a low-pressure furnace, followed by a clean-up treatment of sapphire substrate at $1100^{\circ} \mathrm{C}$. Thereafter, the AlN buffer layer was annealed at a high temperature in the range of $1500^{\circ} \mathrm{C}$ to $1700^{\circ} \mathrm{C}$ for 2 hours under the atmosphere of $\mathrm{N}_{2}+\mathrm{CO}$. The objective of this research is to determine the microstructure changes with different annealing temperatures. Cross-sectional TEM has revealed that, after annealing at $1500^{\circ} \mathrm{C}$, two types of defects remained in the AlN buffer layer: inverted cone shape domains and threading dislocations. The former domains were observed in an image taken with diffraction of $g=0002$, but not in an image with $g=10 \overline{1} 0$. The morphology and the diffraction condition for the image contrast strongly, suggesting that the domains are inversion domains. The threading dislocations were invisible in the image taken with the diffraction of $g=0002$, revealing that they were $a$-type dislocations. However, after annealing at $1600^{\circ} \mathrm{C}$, the inversion domains coalesced with each other to give a two-layer structure divided by a single inversion domain boundary at the centre of the AlN buffer layer. The density of threading dislocation was roughly estimated to be $5 \times 10^{9} \mathrm{~cm}^{-2}$ after annealing at $1500^{\circ} \mathrm{C}$, and to be reduced to $5 \times 10^{8} \mathrm{~cm}^{-2}$ after annealing at $1600^{\circ} \mathrm{C}$. These experimental results validate the fact that the annealing temperature around $1600^{\circ} \mathrm{C}$ is high enough to remove the defects by the diffusion process. Therefore, it was discovered that high temperature annealing is an effective treatment to alter the microstructure of AlN thin films and remove defects by the diffusion process. Annealing at high temperature is recommended to increase the emission efficiency for fabrication of optoelectronic devices.
\end{abstract}

Keywords: Electron microscopy; annealing; aluminium nitride; inversion domain boundary.

\section{INTRODUCTION}

Semiconductor nitrides such as aluminium nitride (AlN), galium nitride $(\mathrm{GaN})$, and aluminium galium nitride ( $\mathrm{AlGaN}$ ) have gained considerable attention over the last two decades as promising materials for optical devices in the blue and UV regions. Due to this potential, semiconductor nitrides have been investigated for application in various photoelectronic devices such as light-emitting diodes (LED), laser diodes (LD) and photo-detectors for the ultraviolet region [1-4]. Aluminium nitride (AlN) specifically has 
a wide band gap $(6.3 \mathrm{eV})$, high decomposition temperature $\left(2490^{\circ} \mathrm{C}\right)$, good dielectric properties and usually crystallizes in wurtzite structure $\left(\mathrm{P}^{3} \mathrm{mc}\right)$ with the lattice parameters of $a=0.311 \mathrm{~nm}$ and $c=0.498 \mathrm{~nm}$, which is the reason why it is extensively studied [5,6]. Considerable interest arose in the last decade in the use of thin films of AlN for various applications, ranging from hard coatings and over-coatings for magneto-optic media to thin film transducers and GHz-band surface acoustic wave devices (SAW) 00010 [7-9]. However, AlN thin film deposited on a sapphire substrate usually contains a lot of lattice defects because of the large lattice mismatch, as well as the poor Al atom migration ability [10]. Therefore, it is very important to establish a method for the growth process to obtain high quality AIN thin films grown on sapphire substrate. In addition, the deposition of an AlN buffer layer reduces the microscopic fluctuation in crystallite orientation and thus improves the crystalline quality. The AlN buffer layer's essential role is thought to be the supply of nucleation centres having the same orientation as the substrate. In addition, the AlN buffer layer promotes the lateral growth of the film, reducing the interfacial free energy between the film and substrate [11, 12]. Apparently, when AlN thin films were grown on a substrate of (0001) sapphire, it was found that the crystallinity of AlN depends upon the growth temperature and the annealing temperature of a buffer layer of AlN deposited on the sapphire substrate. In this work, detailed characterization by electron microscopy was conducted in order to clarify the origin of the heat-treatment dependence, and the changes in microstructure of an AlN buffer layer with annealing at high temperature were investigated in order to clarify the process of the annihilation of lattice defects. Previous research has reported that, as the annealing temperature increases, the surface roughness decreases and forms a granular worm-like nanostructure. The AlN crystalline grains grow larger and reduce the surface roughness monotonically when annealed from $1200^{\circ} \mathrm{C}$ to $1600^{\circ} \mathrm{C}$ for 20 minutes [13]. The impact of post-deposition annealing of sputtered aluminium nitride thin films up to $1000^{\circ} \mathrm{C}$ for 2 hours in nitrogen and oxygen atmosphere results in cracks when the thickness increases, which is due to tensile stress on the island growth between the AlN thin film and substrate. In the same way, after annealing at $1000^{\circ} \mathrm{C}$ for 10 seconds, the threading dislocation density can be reduced from $5 \times 10^{10}$ to $2 \times 10^{9} \mathrm{~cm}^{-2}$. This is evidence that annealing releases the stress in the film and decreases the stress effect on the crystal lattice [14]. AlN annealed at $700^{\circ} \mathrm{C}$ showed improved electrical properties, whereby the flatband voltages $\left(\mathrm{V}_{\mathrm{FB}}\right)$ value was positive and the peak intensity increased [15]. Post-deposition rapid thermal annealing between $900^{\circ} \mathrm{C}$ and $1300^{\circ} \mathrm{C}$ showed a reduction of defects in the AlN thin films' crystallization process and was identified by the increase of grain size of the original crystallites as well as the growth of new small grains exhibiting tensile strain [16].

\section{EXPERIMENTAL}

\section{Growth of Aluminium Nitride}

Researchers have made a number of attempts to grow crystals including single crystals of high quality. Various methods have been reported for the growth of single crystal films including hydride vapour phase epitaxy (HVPE), molecular beam epitaxy (MBE) and metal-organic vapour phase epitaxy (MOVPE) using a sapphire single crystal as a substrate [17-20]. The microstructure and quality of thin films depend strongly on the growth condition. In this current study, an AlN epitaxial layer was grown by the metalorganic vapour phase epitaxy (MOVPE) method in a low-pressure furnace. Trimethylaluminium and ammonia were used as the sources of aluminium and nitrogen 
respectively, together with $\mathrm{H}_{2}$ as carrier gas. As illustrated in Figure 1(a), firstly a c-plane sapphire substrate with a $0.2^{\circ}$ off-angle towards the $m$-direction was cleaned for 10 minutes under $\mathrm{H}_{2}$ atmosphere at a temperature of $1150^{\circ} \mathrm{C}$. Next, an AlN buffer layer of approximately $200 \mathrm{~nm}$ thickness was deposited on a (0001) sapphire substrate at $1150^{\circ} \mathrm{C}$ (MT; medium temperature). After the deposition of the AlN buffer layer, the TMA supply was stopped as the temperature was reduced to $300^{\circ} \mathrm{C}$ by switching the $\mathrm{H}_{2}$ atmosphere with $\mathrm{N}_{2}$ atmosphere to protect the MT-AlN surface, followed by cooling to room temperature by changing back to $\mathrm{H}_{2}$ atmosphere again. After that, the MT-AlN/sapphire template was annealed at $1500^{\circ} \mathrm{C}$ to $1700^{\circ} \mathrm{C}$ for 2 hours in a graphite reaction furnace under a controlled atmosphere of $\left(\mathrm{N}_{2}+\mathrm{CO}\right)$ and the total pressure was 30 Torr during the growth. Finally, an AlN layer was overgrown on the AlN buffer layer with a thickness of 1 micron, as shown in the schematic structure in Figure 1(b).
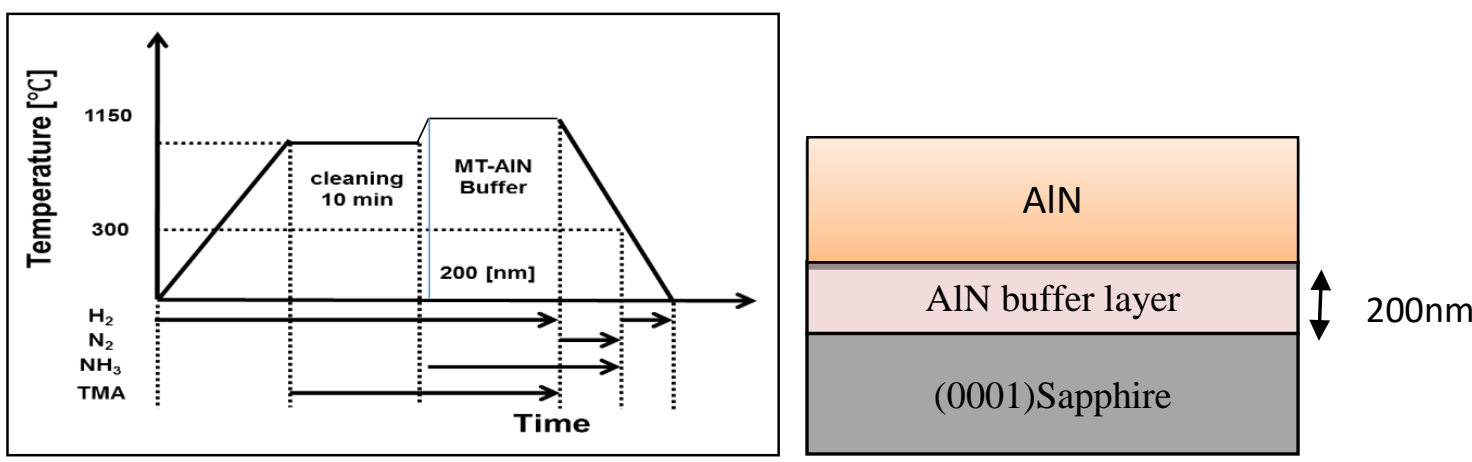

Figure 1(a). Time sequence of AlN growth, (b) Schematic structure of AlN template.
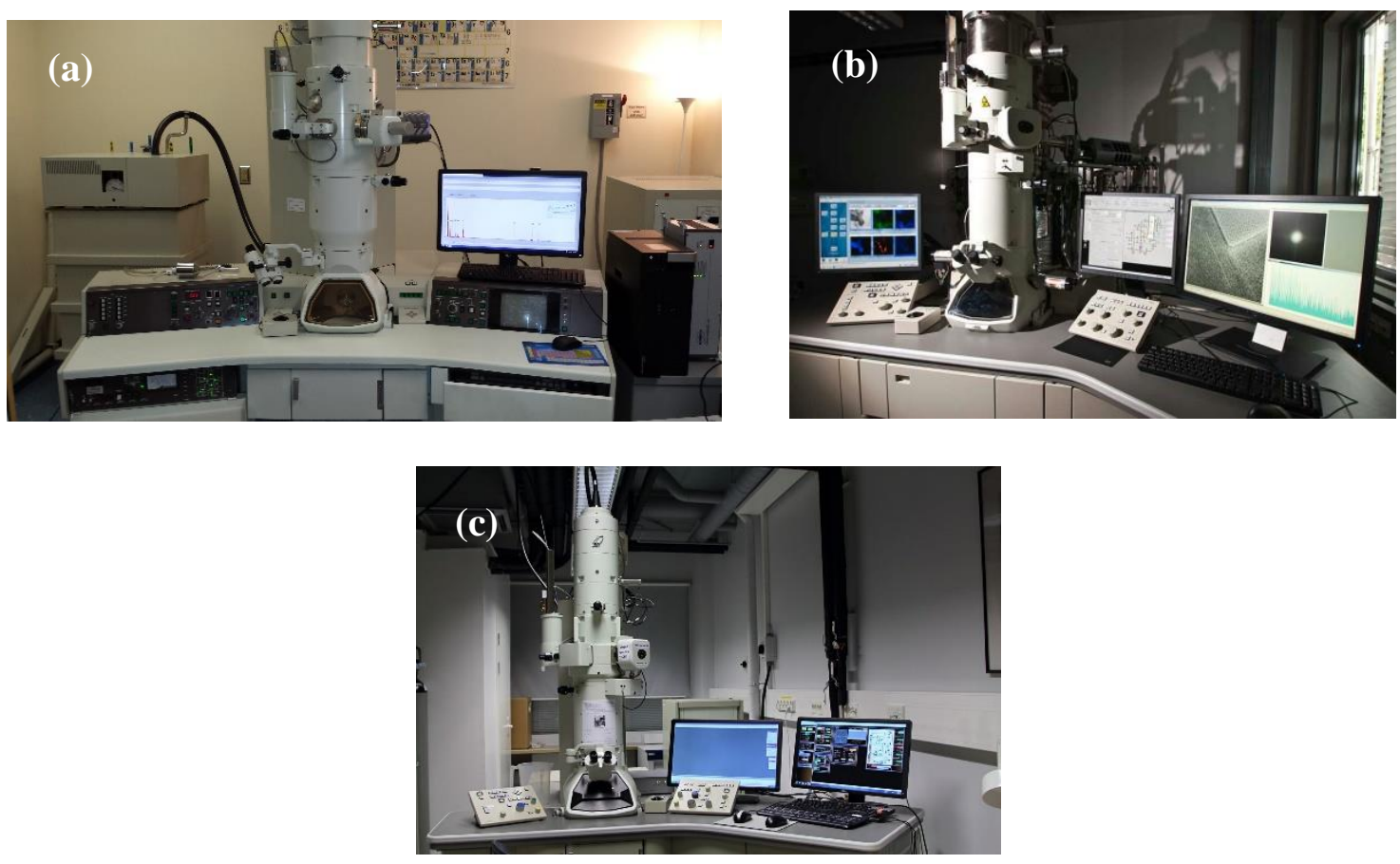

Figure 2. Transmission electron microscope types: (a) JEM-2000EX, (b) JEM 2100, (c) Tecnai-20F (JEOL). 


\section{Characterization}

\section{Transmission Electron Microscopy}

The surface roughness of AlN after annealing at various temperatures was observed with an atomic force microscope (AFM). The root-mean-square (RMS) roughness value was measured. Certainly, to obtain sufficiently large electron transparent areas with a homogeneous orientation, the sample preparation is very crucial and thus has to be carefully conducted [21]. The cross-sectional microstructures and dislocations present in the layers were analysed by a transmission electron microscope (TEM). The microstructure was examined with transmission electron microscope types JEM-2000EX, JEM 2100 and Tecnai-20F (JEOL), as shown in Figure 2(a), (b) and (c) respectively with $200 \mathrm{kV}$ accelerating voltage.
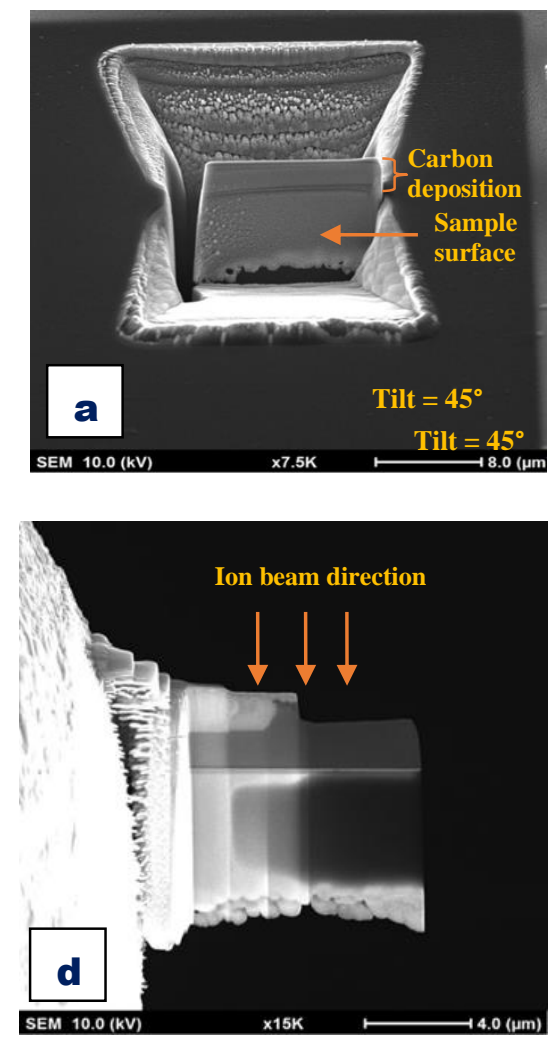
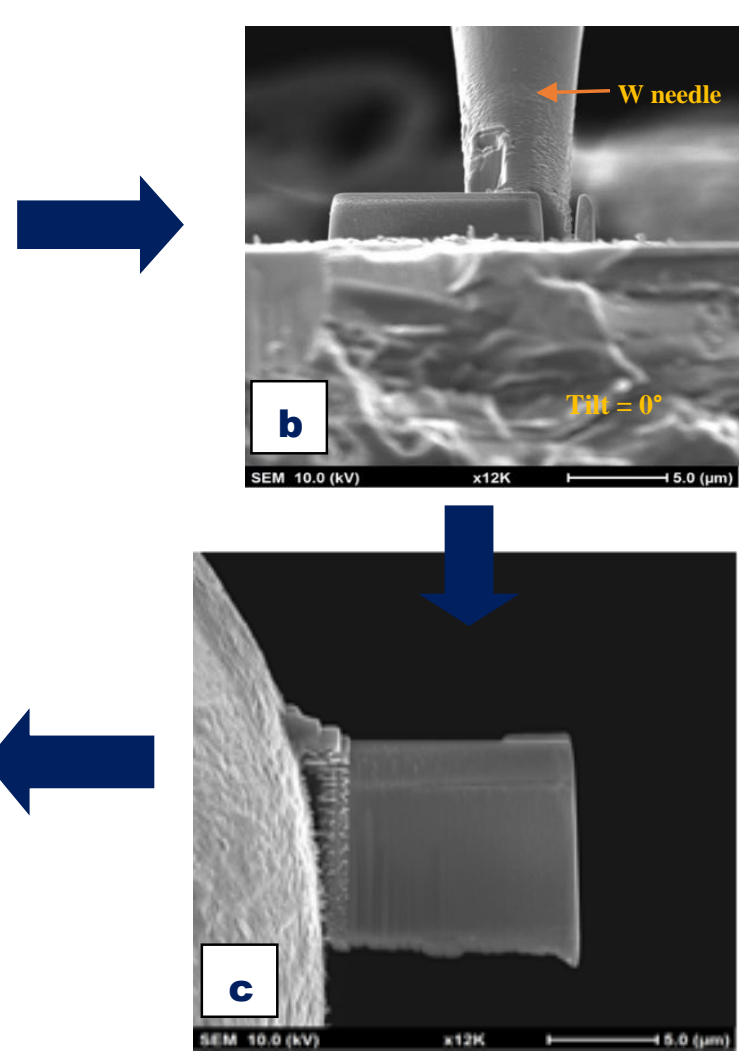

Figure 3. SEM images: (a) specimen coated and dug until $1 \mu \mathrm{m}$ slice remains, (b) slice removed using needle, (c) slice attached to TEM mesh, (d) specimen thinning (15 kV to $3 \mathrm{kV}$ accelerating voltage).

\section{Focused ion beam (FIB) microscopy}

A focused ion beam (FIB) microscope offers both high resolution imaging and flexible micromachining and is used to facilitate the specimen preparation for transmission electron microscopy (TEM) [22]. FIB systems use gallium ions with a low beam current for imaging or a high beam current for sputtering. In the current research, a Hitachi MI4000L dual beam FIB microscope was used to prepare thin foil specimens for TEM observation, followed by finishing with an argon ion mill, whereby the accelerating voltage was decreased from $15 \mathrm{kV}$ to $3 \mathrm{kV}$ to remove damaged layers produced by the FIB milling. Figure 3 explains in brief the step-by-step process for the FIB technique carried out by the researcher for cutting or cross-sectioning nitride semiconductor thin 
films followed by thinning to less than $100 \mathrm{~nm}$ for viewing under the TEM. First the specimen undergoes cutting and is coated before digging. However, it is not cut or etched fully, but only until a $1 \mu \mathrm{m}$ slice remains. Next, a needle is attached to the slice to remove it from the dug hole. After that, the needle attaches the slice to the TEM mesh carefully. Then the slice undergoes a thinning process with a low beam current, followed by argon ion milling for 1 hour to remove the damaged layers as well as point defects produced during the thinning process. Figure 3(a)-(d) shows the step-by-step procedure in specimen preparation for TEM using FIB.

\section{RESULTS AND DISCUSSION}

Figure 4(a) shows the AFM image of the surface morphology of specimens annealed at $1500^{\circ} \mathrm{C}$ and $1600^{\circ} \mathrm{C}$ respectively. As the annealing temperature was increased from $1500^{\circ} \mathrm{C}$ to $1600^{\circ} \mathrm{C}$, the surface morphology, which was initially rough, improved and became atomically flat. The AFM root-mean-square (RMS) roughness decreases monotonously with increased annealing temperature, as presented in Figure 4(b). Likewise, the AFM RMS roughness of the AlN annealed at $1600^{\circ} \mathrm{C}$ was as low as $0.5 \mathrm{~nm}$. As a result, the AFM observation revealed that the grain structure of AlN enlarges with increasing annealing temperature $T_{\mathrm{AN}}$. The atomic steps disappear gradually and the surface becomes smoother. Again, a flat surface morphology is a prerequisite for obtaining smooth and sharp superlattice structures. Consequently, it can be inferred that in the annealing process the AlN film undergoes surface reconstruction, whereby the smaller grains in the epilayer merge into bigger ones, providing a smooth surface. This result is similar to the findings of Miyagawa et al., whereby the surface roughness reduces when the annealing temperature is increased from $1200{ }^{\circ} \mathrm{C}$ to $1600{ }^{\circ} \mathrm{C}[23]$.
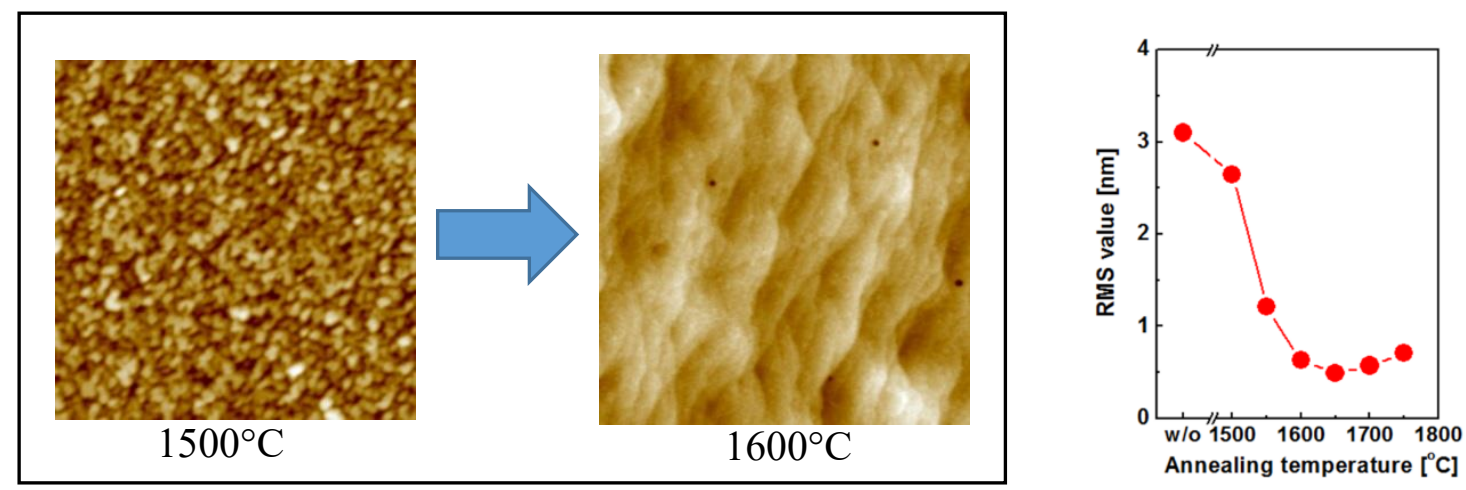

Figure 4. (a) AFM image of AlN annealed at $1500^{\circ} \mathrm{C}$ and $1600^{\circ} \mathrm{C}$, (b) AFM root-meansquare (RMS) vs annealing temperature.

Furthermore, this can be proved with the TEM micrographs taken for both annealing temperatures. Figure 5(a) and (b) shows the TEM micrographs for the bright field image (BF) image and dark field (DF) image taken with the diffraction of $g=0002$ and $g=10 \overline{1} 0$. The BF image shows a lot of inverted cone domains which are similar to overturned ice glaciers. However, these domains are almost invisible due to the weak excitation of the diffraction of $g=10 \overline{1} 0$ and are replaced by a columnar domain. There are a very few threading dislocations (TDs) of c-type and (a+c)-type. TDs of $a$-type are formed but they are out of contrast at the diffraction condition $g=10 \overline{1} 0$. The image contrasts are characteristic of dislocations in two-beam images taken under the condition 
of strong excitation. Due to the absence of threading dislocations in Figure 5(b), the Burgers vectors of the dislocations are considered to be $B=<2-1-10>/ 3$. Threading dislocations are characterized as type-a (edge type), type-c (screw type), and type-a+c (mixed type) according to their Burgers vector. The types of threading dislocations can be distinguished by the visible-invisible criterion in the TEM images. As can be seen in Figure 5(a), threading dislocations of type-c and type- $(\mathrm{a}+\mathrm{c})$ dislocations are visible for $g=0002$. Similarly, type-a and type-a+c dislocations are visible for $g=10 \overline{1} 0$, as shown in Figure 5(b). The estimated dislocation density of the AlN buffer layer is $5 \times 10^{9} \mathrm{~cm}^{-2}$ after annealing at $1500^{\circ} \mathrm{C}$. The narrow columns cone shaped inversion domains extend from the interface to the film surface. The morphology and the diffraction condition for the image contrast strongly, suggesting that the domains are inversion domains [12].

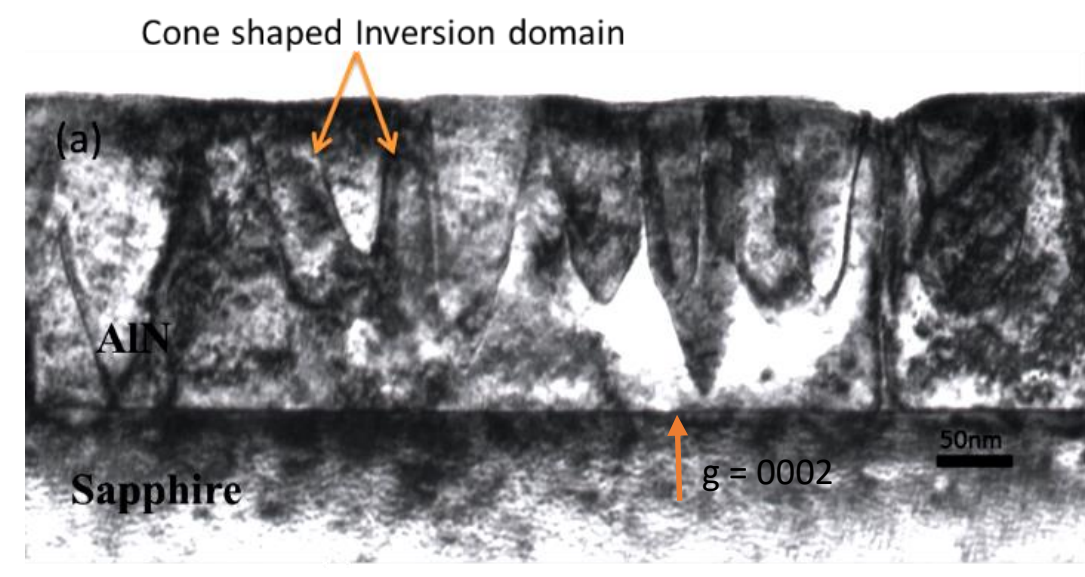

Columnar domain

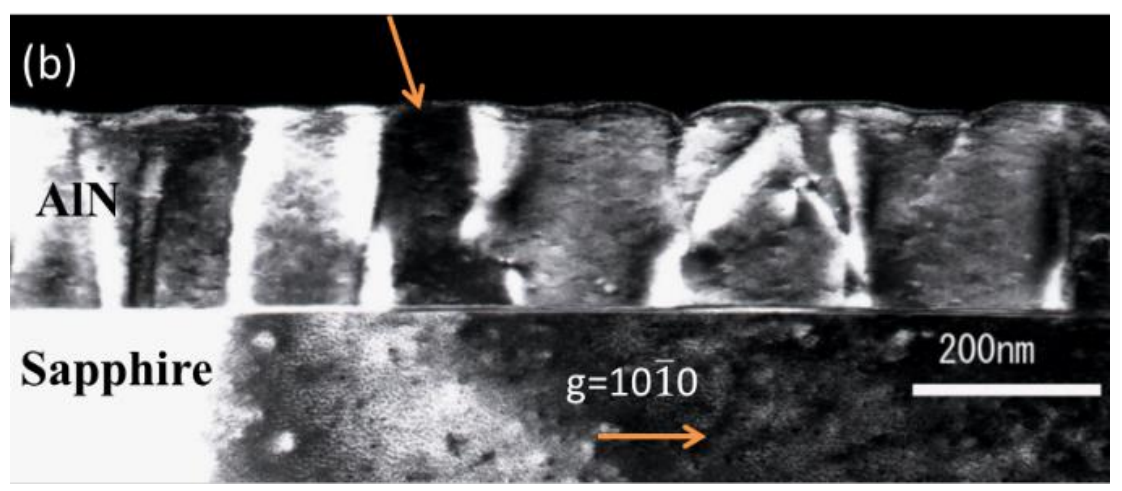

Figure 5. (a) Bright field (BF) image along [1210]0] axis $g=0002$ with $T A N 1500^{\circ} \mathrm{C}$, (b) dark field (DF) image $g=10 \overline{1} 0$ with $T A N 1500^{\circ} \mathrm{C}$.

Figure 6(a) and (b) shows the TEM micrographs of specimens annealed at $1600^{\circ} \mathrm{C}$, whereby the MT-AIN has changed into a two-layer structure. In particular, the inversion domains have coalesced with each other (lateral grain growth process) to leave a single inversion domain boundary running in a zigzag of AlN buffer layer. It is known that in AlN layers inversion domains are frequently formed according to the growth condition. In addition, when the AlN buffer layer is annealed at a temperature of $1600^{\circ} \mathrm{C}$, a smooth grain boundary which separates the AlN layer polarity is observed. The grain boundary is no longer at the centre of the AIN thin film buffer layer. The grain boundary has become lower and certain parts are even touching the interface between the AlN buffer layer and 
sapphire substrate. The Al polarity area is increasing compared to the $\mathrm{N}$ polarity area. We can also observe a threading dislocation line intersecting the inversion domain grain boundary, as shown in the BF image in Figure 6(a).On the other hand, the subgrain boundary is invisible instead of the c-type threading dislocation from the interface of the AlN and sapphire, moving upwards and bending at the surface of the film as shown in Figure 6(b). The dislocation density has also been reduced to $5 \times 10^{8} \mathrm{~cm}^{-2}$ after annealing at $1600^{\circ} \mathrm{C}$. It has been confirmed that annealing can be an effective treatment to control the microstructure of AlN thin films and further reduce the dislocations present in the specimen. In short, when the dislocations are reduced, the thin film efficiency is increased. Further research is ongoing to determine the crystallographic polarity, including high-resolution CTEM, convergent electron beam diffraction (CBED), atomlocation by channelling-enhanced micro-analysis (ALCHEMI) with energy dispersive $\mathrm{x}$ ray spectroscopy (EDS) or electron energy loss spectroscopy (EELS) [24]. The CBED method can show direct evidence of the polarity of $\mathrm{GaN}$ under the conventional condition of an accelerating voltage of $200 \mathrm{kV}$, as reported by Adachi et al. [25].
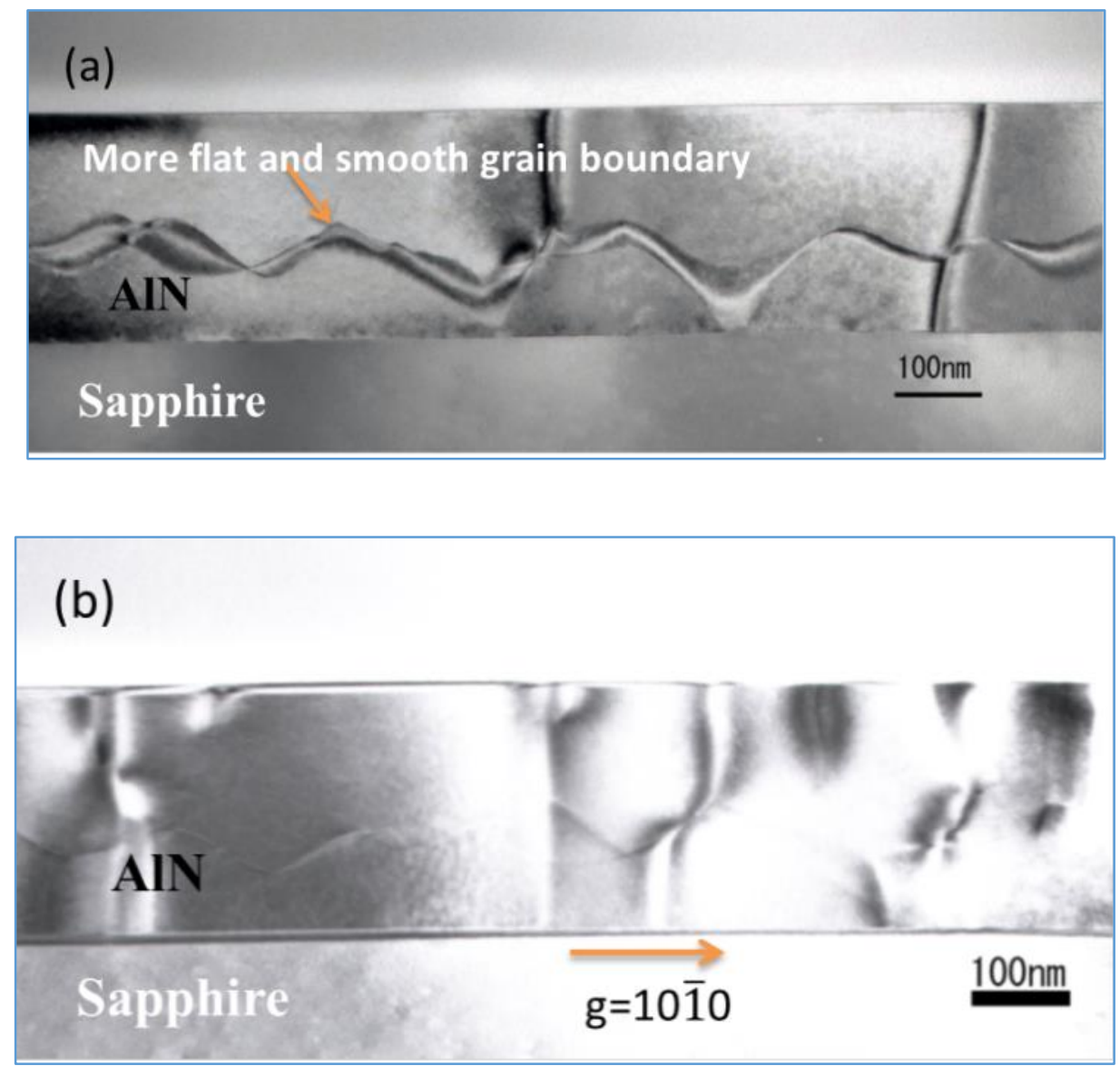

Figure 6. (a) Bright field (BF) image along [ $\overline{1} 2 \overline{1} 0]$ axis $g=0002$ with $T A N 1600^{\circ} \mathrm{C}$, (b) BF image $g=10 \overline{1} 0$ with $T A N 1600^{\circ} \mathrm{C}$.

\section{CONCLUSIONS}

The effects of annealing temperature on the microstructure of AlN buffer layer were investigated by AFM and TEM. The following results were obtained: 
i). The AlN layer contains many inversion domains of inverted cone shape. During annealing, the inversion domains coalesce with each other to form a two-layer structure divided by a single inversion domain boundary.

ii). The inversion domain boundary runs laterally in a zigzag with sharp corners in the AlN layer.

iii). The inversion domain boundary becomes flatter and smoother with annealing at a higher temperature.

iv). The annealing temperature around $1600^{\circ} \mathrm{C}$ is high enough to change the microstructure by a diffusion process.

v). It has been confirmed that annealing can be an effective treatment to control the microstructure of AlN thin films.

\section{ACKNOWLEDGEMENTS}

The authors would like to express their gratitude to Malaysia-Japan International Institute of Technology, UTM for providing laboratory facilities and financial assistance under Fundamental Research Grant Scheme (FRGS/2/2013/SG06/UTM/01/2) from Ministry of Education (MOE), Malaysia. This work was supported in part and the present authors are grateful for technical supports from Nanotechnology Platform Japan (Kyushu University), Japan.

\section{REFERENCES}

[1] Akasaki I, Amano H. Crystal growth and conductivity control of group III nitride semiconductors and their application to short wavelength light emitters. Japanese Journal of Applied Physics. 1997;36:5393.

[2] Ito H. Generation-recombination current in the emitter-base junction of AlGaAs/GaAs HBTs. Japanese Journal of Applied Physics. 1986;25:1400.

[3] Mukai T, Morita D, Nakamura S. High-power UV InGaN/AlGaN doubleheterostructure LEDs. Journal of Crystal Growth. 1998;189-190:778-81.

[4] Ponce F, Srinivasan S, Bell A, Geng L, Liu R, Stevens M, et al. Microstructure and electronic properties of InGaN alloys. Physica Status Solidi (Basic Solid State Physics). 2003;240:273-84.

[5] Rodriguez-Navarro A, Otañno-Rivera W, Garcia-Ruiz J, Messier R, Pilione L. Preparation of highly oriented polycrystalline AlN thin films deposited on glass at oblique-angle incidence. Journal of Materials Research. 1997;12:1689-92.

[6] Nishida T, Makimoto T, Saito H, Ban T. AlGaN-based ultraviolet light-emitting diodes grown on bulk AlN substrates. Appl Phys Lett. 2004;84:1002-3.

[7] Rubio-Roy M, Corbella C, Bertran E, Andújar JL. Tribological properties of fluorinated amorphous carbon thin films: INTECH Open Access Publisher; 2011.

[8] Nomura T, Okumura K, Miyake H, Hiramatsu K, Eryu O, Yamada Y. AlN homoepitaxial growth on sublimation-AIN substrate by low-pressure HVPE. Journal of Crystal Growth. 2012;350:69-71.

[9] Okano H, Tanaka N, Takahashi Y, Tanaka T, Shibata K, Nakano S. Preparation of aluminum nitride thin films by reactive sputtering and their applications to GHz-band surface acoustic wave devices. Applied Physics Letters. 1994;64:1668. 
[10] Pei-Qiang X, Yang J, Zi-Guang M, Zhen D, Tai-Ping L, Chun-Hua D, et al. The influence of graded AlGaN buffer thickness for crack-free GaN on Si (111) substrates by using MOCVD. Chinese Physics Letters. 2013;30:028101.

[11] Imura M, Nakano K, Fujimoto N, Okada N, Balakrishnan K, Iwaya M, et al. Dislocations in AIN epilayers grown on sapphire substrate by high-temperature metal-organic vapor phase epitaxy. Japanese Journal of Applied Physics. 2007;46:1458.

[12] Adachi M, Tsuda K, Sugiyama M, Iida J, Tanaka A, Fukuyama H. High-quality AlN layer homoepitaxially grown on nitrided a-plane sapphire using a GA? Al Flux. Applied Physics Express. 2013;6:091001.

[13] Wei-Ying W, Peng J, Gui-Peng L, Wei L, Bin L, Xing-Fang L, et al. Effect of high-temperature annealing on AlN thin film grown by metalorganic chemical vapor deposition. Chinese Physics B. 2014;23:087810.

[14] Liu B, Gao J, Wu K, Liu C. Preparation and rapid thermal annealing of AlN thin films grown by molecular beam epitaxy. Solid State Communications. 2009;149:715-7.

[15] Gillinger M, Schneider M, Bittner A, Nicolay P, Schmid U. Impact of annealing temperature on the mechanical and electrical properties of sputtered aluminum nitride thin films. Journal of Applied Physics. 2015;117:065303.

[16] Hu Y-M, Li S-S, Kuang C-H, Han T-C, Yu C-C. Post-annealing effect on the room-temperature ferromagnetism in $\mathrm{Cu}$-doped $\mathrm{ZnO}$ thin films. Journal of Applied Physics. 2015;117:17B901.

[17] Kuech T. Metal-organic vapor phase epitaxy of compound semiconductors. Materials Science Reports. 1987;2:1-49.

[18] Liu B, Fu Q, Wu K, Liu C. Studies of the growth method and properties of AIN grown by RF-MBE. Journal of the Korean Physical Society. 2008;52.

[19] Richter E, Hennig C, Weyers M, Habel F, Tsay J-D, Liu W-Y, et al. Reactor and growth process optimization for growth of thick $\mathrm{GaN}$ layers on sapphire substrates by HVPE. Journal of Crystal Growth. 2005;277:6-12.

[20] Hemmingsson C, Paskov P, Pozina G, Heuken M, Schineller B, Monemar B. Hydride vapour phase epitaxy growth and characterization of thick GaN using a vertical HVPE reactor. Journal of Crystal Growth. 2007;300:32-6.

[21] Reyntjens S, Puers R. A review of focused ion beam applications in microsystem technology. Journal of Micromechanics and Microengineering. 2001;11:287.

[22] Williams DB, Carter CB. The transmission electron microscope. Transmission electron microscopy: Springer; 1996. p. 3-17.

[23] Miyagawa R, Yang S, Miyake H, Hiramatsu K, Kuwahara T, Mitsuhara M, et al. Microstructure of AlN grown on a nucleation layer on a sapphire substrate. Applied Physics Express. 2012;5:025501.

[24] Imura M, Gautam U, Nakajima K, Koide Y, Amano H, Tsuda K. Analysis of broken symmetry in convergent-beam electron diffraction along $\left\langle 11_{2} 0\right\rangle$ and $\left.<1_{1} 00\right\rangle$ zone-axes of AlN for polarity determination. Japanese Journal of Applied Physics. 2013;52:08JE15.

[25] Adachi M, Takasugi M, Morikawa D, Tsuda K, Tanaka A, Fukuyama H. Analysis of the dislocation and polarity in an AlN layer grown using GA-Al flux. Applied Physics Express. 2012;5:101001. 